Pacific Journal of Mathematics

CYCLIC GROUPS OF AUTOMORPHISMS OF COMPACT
NONORIENTABLE KLEIN SURFACES WITHOUT BOUNDARY 


\title{
CYCLIC GROUPS OF AUTOMORPHISMS OF COMPACT NON-ORIENTABLE KLEIN SURFACES WITHOUT BOUNDARY
}

\author{
EMilio BuJALANCE
}

\begin{abstract}
We obtain the minimum genus of the compact non-orientable Klein surfaces of genus $p \geq 3$ without boundary which have a given cyclic group of automorphisms.
\end{abstract}

1. Introduction. Let $X$ be a compact Klein surface [1]. Singerman [8] showed that the order of a group of automorphisms of a surface $X$ without boundary of algebraic genus $g \geq 2$ is bounded above by $84(g-1)$, and May [7] proved that if $X$ has nonempty boundary, this bound is $12(g-1)$.

These bounds may be considered as particular cases of the general problem of finding the minimum genus of surfaces for which a given finite group $G$ is a group of automorphisms. The study of cyclic groups is a necessary preliminary to this, since it leads to limitations on the orders of elements within a general group. In this paper we consider the above problem for the case of cyclic groups of automorphisms of compact non-orientable Klein surfaces without boundary. The corresponding problem for compact orientable Klein surfaces without boundary was solved by Harvey [5].

2. Compact non-orientable Klein surfaces without boundary. By a non-Euclidean crystallographic (NEC) group, we shall mean a discrete subgroup $\Gamma$ of the group of isometries $G$ of the non-Euclidean plane, with compact quotient space, including those which reverse orientation, reflections and glide reflections. We say that $\Gamma$ is a proper NEC group if it is not a Fuchsian group. We shall denote by $\Gamma^{+}$the Fuchsian group $\Gamma \cap G^{+}$, where $G^{+}$is the subgroup of $G$ whose elements are the orientation-preserving isometries.

NEC groups are classified according to their signature. The signature of an NEC group $\Gamma$ is either of the form

$$
\left(g ;+;\left[m_{1}, \ldots, m_{\tau}\right] ; \quad\left\{\left(n_{i 1}, \ldots, n_{l s_{l}}\right)_{i=1, \ldots, k}\right\}\right)
$$

or

$(* *)$

$$
\left(g ;-;\left[m_{1}, \ldots, m_{\tau}\right] ; \quad\left\{\left(n_{i 1}, \ldots, n_{\iota s_{i}}\right)_{l=1, \ldots, k}\right\}\right)
$$


the numbers $m_{l}$ are the periods and the brackets $\left(n_{t 1}, \ldots, n_{t s_{l}}\right)$, the period cycles.

A group $\Gamma$ with signature $(*)$ has the presentation given by generators

$$
\begin{gathered}
x_{\imath}, \quad i=1, \ldots, \tau, \quad c_{\imath j}, \quad i=1, \ldots, k, j=0, \ldots, s_{\imath}, \\
e_{\imath}, \quad i=1, \ldots, k, \quad a_{J}, b_{j}, j=1, \ldots, g,
\end{gathered}
$$

and relations

$$
\begin{gathered}
x_{l}^{m_{\imath}}=1, \quad i=1, \ldots, \tau, \quad c_{l s_{l}}=e_{l}^{-1} c_{\imath 0} e_{l}, \quad i=1, \ldots, k, \\
c_{l j-1}^{2}=c_{l j}^{2}=\left(c_{l j-1} \cdot c_{i j}\right)^{n_{\imath \jmath}}=1, \quad i=1, \ldots, k, j=1, \ldots, s_{l}, \\
x_{1} \cdots x_{\tau} e_{1} \cdots e_{k} a_{1} b_{1} a_{1}^{-1} b_{1}^{-1} \cdots a_{g} b_{g} a_{g}^{-1} b_{g}^{-1}=1 .
\end{gathered}
$$

A group $\Gamma$ with signature $(* *)$ has the presentation given by generators

$$
\begin{gathered}
x_{\imath}, \quad i=1, \ldots, \tau, \quad c_{\imath j}, \quad i=1, \ldots, k, j=0, \ldots, s_{\imath}, \\
e_{l}, \quad i=1, \ldots, k, \quad d_{j}, \quad j=1, \ldots, g,
\end{gathered}
$$

and relations

$$
\begin{gathered}
c_{l s_{t}}=e_{l}^{-1} c_{\imath 0} e_{\imath}, \quad i=1, \ldots, k, \quad x_{\imath}^{m_{\imath}}=1, \quad i=1, \ldots, \tau, \\
c_{\imath,-1}^{2}=c_{l J}^{2}=\left(c_{\imath \jmath-1} \cdot c_{i j}\right)^{n_{\imath \jmath}}=1, \quad i=1, \ldots, k, j=1, \ldots, s_{i}, \\
x_{1} \cdots x_{\tau} e_{1} \cdots e_{k} d_{1}^{2} \cdots d_{g}^{2}=1 .
\end{gathered}
$$

From now on, we will denote by $x_{i}, e_{l}, c_{i j}, a_{i}, b_{l}, d_{l}$ the above generators associated to the NEC groups.

(2.1) Definition. We shall say that an NEC group $\Gamma_{g}$ is the group of an orientable surface if $\Gamma_{g}$ has the signature $(g ;+;[-] ;\{-\})$ where $[-]$ indicates that the signature has no periods and $\{-\}$ indicates that the signature has no period cycles.

(2.2) Definition. An NEC group $\Gamma_{p}$ is the group of a non-orientable surface if $\Gamma_{p}$ has the following signature $(p ;-;[-] ;\{-\})$.

For a given $\Gamma_{p}$ we have that the orbit space $D / \Gamma_{p}$ (where $D=C^{+}$) is a non-orientable surface of genus $p$. The canonical projection $\pi: D \rightarrow D / \Gamma_{p}$ induces an analytic and anti-analytic structure on $D / \Gamma_{p}$, which establishes a structure of compact non-orientable Klein surface without boundary of genus $p$ in $D / \Gamma_{p}$. 
From now on, Klein surfaces appearing in this paper are supposed to be compact without boundary.

Singerman has shown in $[8]$ the following

(2.3) Proposition. If $G$ is a group of automorphisms of a non-orientable Klein surface of genus $p \geq 3$, then $G$ is finite.

(2.4) THEOREM. A necessary and sufficient condition for a finite group $G$ to be a group of automorphisms of a non-orientable Klein surface of genus $p \geq 3$ is that there exist a proper NEC group $\Gamma$ and a homomorphism $\theta$ : $\Gamma \rightarrow G$ such that the kernel of $\theta$ is a surface group and $\theta\left(\Gamma^{+}\right)=G$.

As a consequence of this theorem, we have that if $G$ is a finite group of automorphisms of a non-orientable Klein surface of genus $p \geq 3$ then $G \simeq \Gamma / \Gamma_{p}$, where $\Gamma$ is a proper NEC group and $\Gamma_{p}$ is the group of a non-orientable surface; thus

$$
\operatorname{order}(G)=\left|\Gamma_{p}\right| /|\Gamma|=2 \pi(p-2) /|\Gamma|,
$$

where || denotes the non-Euclidean area of a fundamental region of the group.

(2.5) THEOREM. If $G$ is a finite group, $G$ is a group of automorphisms of a non-orientable Klein surface of genus $p \geq 3$.

Proof. Let us suppose that $G$ has $n$ generators $g_{1}, g_{2}, \ldots, g_{n}$. There exists a proper NEC group $\Gamma_{2 n+1}$ that is the group of a non-orientable surface, and therefore it has the following generators and relations:

$$
\left\{a_{1}, a_{2}, \ldots, a_{2 n+1} \mid a_{1}^{2} \cdot a_{2}^{2} \cdots a_{2 n+1}^{2}=1\right\} .
$$

We establish a homomorphism $\theta: \Gamma_{2 n+1} \rightarrow G$, by defining

$$
\begin{gathered}
\theta\left(a_{1}\right)=g_{1}, \quad \theta\left(a_{3}\right)=g_{2} \cdots \theta\left(a_{2 n-1}\right)=g_{n}, \quad \theta\left(a_{2 n+1}\right)=1, \\
\theta\left(a_{2}\right)=g_{1}^{-1}, \quad \theta\left(a_{4}\right)=g_{2}^{-1}, \quad \theta\left(a_{2 n}\right)=g_{n}^{-1} .
\end{gathered}
$$

$\theta$ is an epimorphism. $\operatorname{ker} \theta$ is a normal subgroup of $\Gamma_{2 n+1}$ with finite index, and therefore, $\operatorname{ker} \theta$ is an NEC group.

As $\Gamma_{2 n+1}$ has neither periods nor period-cycles, and $\operatorname{ker} \theta$ is a normal subgroup of $\Gamma_{2 n+1}$, by [2] and [3], $\operatorname{ker} \theta$ has neither periods nor periodcycles, and thus it is a surface group.

Moreover, as $a_{1} \cdot a_{2 n+1}, a_{3} \cdot a_{2 n+1}, \ldots, a_{2 n-1} \cdot a_{2 n+1}$ belong to $\Gamma_{2 n+1}^{+}$ and $\theta\left(a_{1} \cdot a_{2 n+1}\right)=g_{1}, \quad \theta\left(a_{3} \cdot a_{2 n+1}\right)=g_{2}, \ldots, \theta\left(a_{2 n-1} \cdot a_{2 n+1}\right)=g_{n}$, then $\theta\left(\Gamma_{2 n+1}^{+}\right)=G$. 
By (2.4) $G$ is a group of automorphisms of a non-orientable Klein surface of genus $p \geq 3$.

\section{Non-orientable surface-kernel homomorphisms.}

(3.1) Definition. A homomorphism $\theta$ of a proper NEC group $\Gamma$ into a finite group is a non-orientable surface-kernel homomorphism if $\operatorname{ker} \theta$ is the group of a surface and $\theta\left(\Gamma^{+}\right)=G$.

From [2], [3] and (2.4) we get

(3.2) Proposition. A homomorphism $\theta$ of a proper NEC group $\Gamma$ of signature $\left(g ; \pm ;\left[m_{1}, \ldots, m_{\tau}\right] ;\left\{\left(n_{11}, \ldots, n_{1 s_{1}}\right) \ldots\left(n_{k 1}, \ldots, n_{k s_{k}}\right)\right\}\right)$ into a finite group $G$ is a non-orientable surface-kernel homomorphism if and only if $\theta\left(c_{l j}\right)$ has order $2, \theta\left(x_{l}\right)$ has order $m_{l}, \theta\left(c_{i,-1} \cdot c_{l j}\right)$ has order $n_{l,}$ and $\theta\left(\Gamma^{+}\right)=G$.

(3.3) COROLlaRY. Let $G$ be a finite group with odd order. Then there is no proper NEC group $\Gamma$ with period cycles for which there exists a non-orientable surface-kernel homomorphism $\theta: \Gamma \rightarrow G$.

(3.4) COROLlaRY. There does not exist any proper NEC group $\Gamma$ with period cycles having some non-empty period cycle for which there is a non-orientable surface-kernel homomorphism $\theta: \Gamma \rightarrow Z_{n}$ with $n$ even.

Proof. If there were a non-orientable surface-kernel homomorphism $\theta: \Gamma \rightarrow Z_{n}$, we would have that for every $c_{l} \in \Gamma, \theta\left(c_{l}\right)$ would have order 2 in $Z_{n}$; if $\Gamma$ has some non-empty period cycle, there would be two reflections $c_{\imath \jmath}, c_{\imath \jmath+1} \in \Gamma$ such that $\left(c_{\imath \jmath} \cdot c_{\imath \jmath+1}\right)^{n_{t \jmath}}=1$ and, by (3.2), the order of $\theta\left(c_{l,} \cdot c_{\imath \jmath+1}\right)$ would be $n_{l}$, but this is impossible because

$$
\theta\left(c_{i j} \cdot c_{\imath \jmath+1}\right)=\theta\left(c_{i \jmath}\right)+\theta\left(c_{i j+1}\right)=\bar{n} / 2+\bar{n} / 2=\bar{n},
$$

where $\bar{p}$ denotes the equivalence class of the element $p$ of $Z_{n}$.

(3.5) THEOREM. Let $\Gamma$ be a proper NEC group with signature

$$
\left(g ;+;\left[m_{1}, \ldots, m_{\tau}\right] ;\{(-)(-), \ldots,(-)\}\right)
$$

and let $n$ be even. Then there exists a non-orientable surface-kernel homomorphism $\theta: \Gamma \rightarrow Z_{n}$ if and only if:

(i) $m_{1} \backslash n \forall i \in I, I=\{1, \ldots, \tau\}$;

(ii) if $g=0, k=1$, then 1.c.m. $\left(m_{1} \cdots m_{\tau}\right)=n$. 
Proof. If there is a non-orientable surface-kernel homomorphism $\theta$ : $\Gamma \rightarrow Z_{n}$, then, by (3.2), $\theta\left(\Gamma^{+}\right)=Z_{n}$.

By Theorem 2 of [9] and Theorem 4 of [5], (i) and (ii) hold.

If we suppose that the elements of the signature $\Gamma$ fulfill (i) and (ii), we define the homomorphism $\theta: \Gamma \rightarrow Z_{n}$ in the following way:

if $g \neq 0$ :

$$
\begin{gathered}
\theta\left(a_{1}\right)=\overline{1}, \quad \theta\left(a_{1}\right)=\bar{n}, \quad i=2, \ldots, g, \quad \theta\left(x_{\imath}\right)=\frac{\bar{n}}{m_{l}}, \\
\theta\left(b_{1}\right)=\overline{1}, \quad \theta\left(b_{i}\right)=\bar{n}, \quad \theta\left(c_{i}\right)=\frac{\bar{n}}{2}, \\
\theta\left(e_{1}\right)=-\overline{\sum_{i=1}^{\tau} \frac{n}{m_{i}}}, \quad \theta\left(e_{1}\right)=\bar{n}, \quad i=2, \ldots, k ;
\end{gathered}
$$

if $g=0, k=1$ :

$$
\theta\left(x_{i}\right)=\frac{\bar{n}}{m_{l}}, \quad \theta\left(c_{j}\right)=\frac{\bar{n}}{2}, \quad \theta\left(e_{1}\right)=-\overline{\sum_{i=1}^{\tau} \frac{n}{m_{l}}} ;
$$

if $g=0, k>1$ :

$$
\begin{gathered}
\theta\left(x_{i}\right)=\frac{\bar{n}}{m_{i}}, \quad \theta\left(c_{l}\right)=\frac{\bar{n}}{2}, \quad \theta\left(e_{1}\right)=\overline{1}, \\
\theta\left(c_{2}\right)=-1-\sum_{i=1}^{\tau} \frac{n}{m_{i}}, \quad \theta\left(e_{l}\right)=\bar{n}, \quad i=3, \ldots, k ;
\end{gathered}
$$

in every case there is a $\gamma \in \Gamma^{+}$such that $\theta(\gamma)=\overline{1}$ :

if $g \neq 0, \gamma=a_{1}$;

if $g=0, k=1$, by (ii) 1.c.m. $\left(m_{1} \cdots m_{\tau}\right)=n$, for there exist integers $\alpha_{1}, \ldots, \alpha_{\tau}$ such that $\alpha_{1} n / m_{1}+\cdots+\alpha \tau n / m_{\tau}=1$, therefore $\gamma=$ $x_{1}^{\alpha_{1}} \cdots x_{\tau}^{\alpha_{r}}$

if $g=0, k>1, \gamma=e_{1}$.

Therefore $\theta\left(\Gamma^{+}\right)=Z_{n}$ and $\theta$ is a non-orientable surface-kernel homomorphism.

(3.6) THEOREM. Let $\Gamma$ be a proper NEC group of signature

$$
\left(g ;-;\left[m_{1}, \ldots, m_{\tau}\right] ;\{(-)(-), \ldots,(-)\}\right)
$$

and let $\theta$ be a non-orientable surface-kernel homomorphism $\theta: \Gamma \rightarrow Z_{n}$ with $n$ even. Then

(i) $m_{\imath} \backslash n \forall i \in I, I=\{1,2, \ldots, \tau\}$;

(ii) if $g=1, k=0$, then l.c.m. $\left(m_{1} \cdots m_{\tau}\right)=n$. 
Proof. The Conditions (i) and (ii) hold by Theorem 2 of [9] and Theorem 4 of [5].

(3.7) THEOREM. Let $\Gamma$ be a proper NEC group of signature ( $g$; - ; $\left.\left[m_{1} \cdots m_{\tau}\right]\right)$ and let $n$ be odd. Then there exists a non-orientable surfacekernel homomorphism $\theta: \Gamma \rightarrow Z_{n}$ if and only if

(i) $m_{i} \backslash n \forall i \in I, I=\{1, \ldots, \tau\}$;

(ii) if $g=1$, then 1.c.m. $\left(m_{1} \cdots m_{\tau}\right)=n$.

Proof. The necessity is similar to (3.6). Let us see the sufficiency. If we suppose that the elements of $\Gamma$ fulfill (i) and (ii) we define the homomorphism $\theta: \Gamma \rightarrow Z_{n}$ in the following way: assume $\overline{\sum_{i \in I} n / m_{l}}=\bar{p}$.

If $g=1$ and $p$ odd:

$$
\theta\left(x_{i}\right)=\frac{\bar{n}}{m_{i}}, \quad \theta\left(a_{1}\right)=\overline{\frac{1}{2}(n-p)} .
$$

If $g=1$ and $p$ even:

$$
\theta\left(x_{\imath}\right)=\frac{\bar{n}}{m_{i}}, \quad \theta\left(a_{1}\right)=\overline{-\frac{1}{2} p} .
$$

If $g>1$ and $p$ odd:

$$
\begin{array}{ll}
\theta\left(x_{i}\right)=\frac{\bar{n}}{m_{i}}, & \theta\left(a_{2}\right)=\frac{\overline{n-2 p-1}}{2}, \\
\theta\left(a_{1}\right)=\frac{\overline{p+1}}{2}, & \theta\left(a_{i}\right)=\bar{n}, \quad i>2 .
\end{array}
$$

If $g>1$ and $p$ even:

$$
\begin{array}{ll}
\theta\left(x_{1}\right)=\frac{\bar{n}}{m_{i}}, & \theta\left(a_{2}\right)=\frac{\overline{n+1}}{2}, \\
\theta\left(a_{1}\right)=\frac{\overline{-p-n-1}}{2}, & \theta\left(a_{i}\right)=\bar{n}, \quad i>3 .
\end{array}
$$

In every case there is $\gamma \in \Gamma^{+}$such that $\theta(\gamma)=\overline{1}$ : if $g=1$, by (ii) 1.c.m. $\left(m_{1} \cdots m_{\tau}\right)=n$, for there exist integers $\alpha_{1}, \ldots, \alpha_{\tau}$ such that $\alpha_{1} n / m_{1}$ $+\cdots+\alpha_{\tau} n / m_{\tau}=1$, therefore $\gamma=x_{1}^{\alpha_{1}} \cdots x_{\tau}^{\alpha_{\tau}}$;

if $g>1$ and $p$ odd, $\gamma=a_{1}^{4} \cdot a_{2}^{2}$;

if $g>1$ and $p$ even, $\gamma=a_{2}^{2}$.

Therefore $\theta\left(\Gamma^{+}\right)=Z_{n}$ and $\theta$ is a non-orientable surface-kernel homomorphism. 
4. Minimum genus. In this section we shall compute the minimum genus of a non-orientable Klein surface which has a cyclic group of automorphisms. We know by (2.4) that if $G$ is a group of automorphisms of a non-orientable Klein surface of genus $p \geq 3$, then $G \simeq \Gamma / \Gamma_{p}$, where $\Gamma$ is a proper NEC group, and $\Gamma_{p}$ is a group of a non-orientable surface. Thus if $\operatorname{order}(G)=n$, we have

$$
n=2 \pi(p-2) /|\Gamma|
$$

and $p=2+(n / 2 \pi)|\Gamma|$, so we can reduce the problem to the search of a proper NEC group for which there exists a non-orientable surface-kernel homomorphism $\theta: \Gamma \rightarrow Z_{n}$ which minimizes $p$.

(4.1) THEOREM. If $n=1, q$ prime, then the minimum genus $p$ of a non-orientable Klein surface with a group of automorphisms isomorphic to $Z_{n}$ is:

$$
\begin{array}{ll}
\text { if } q=2, & p=3 \\
\text { if } q \neq 2, & p=q .
\end{array}
$$

Proof. If $q=2$, we consider an NEC group of signature

$$
(0 ;+;[2,2,2] ;\{(-)\}) \text {. }
$$

This group fulfills the conditions of Theorem (3.5), so

$$
(p-2) / 2=1 / 2 \text {, i.e. } p=3 \text {. }
$$

If $q \neq 2$, we have that an NEC group of signature $(1 ;-;[q, q])$ fulfills the conditions of Theorem (3.7), therefore it is the group of a surface and

$$
(p-2) / q=1-2 / q, \text { i.e. } p=q .
$$

Now let us see that $q$ is the minimum genus.

If we take any other NEC group $\Gamma$ with the conditions of Theorem (3.7), $\Gamma$ would have the signature $(g ;-;[q, \ldots, q])$ and

$$
\begin{gathered}
\frac{p-2}{q}=g-2+\tau\left(1-\frac{1}{q}\right)=(\tau+g-2)-\frac{\tau}{q}, \\
p=2+(\tau+g-2) q-\tau,
\end{gathered}
$$

since $\tau>1$ if $g=1$, and $g \geq 1$, then the following expression is always $\geq q$. 
(4.2) THEOREM. If $n=2^{\beta} q_{1}^{r_{1}} \cdots q_{\alpha}^{r_{\alpha}}$, where $2<q_{1}<\cdots<q_{\alpha}$ and $q_{1}$ $\cdots q_{\alpha}$ are prime, then the minimum genus $p$ of a non-orientable Klein surface with group of automorphisms isomorphic to $Z_{n}$ is

$$
\begin{array}{ll}
p=n / 2 & \text { if } \beta=1, \\
p=n / 2+1 & \text { if } \beta>1 .
\end{array}
$$

Proof. If $\beta=1$, we consider an NEC group $\Gamma$ of signature

$$
(0 ;+;[2, n / 2] ;\{(-)\}) \text {. }
$$

This group fulfills the conditions of Theorem (3.5), so

$$
\frac{p-2}{n}=\frac{1}{2}-\frac{2}{n}, \text { i.e. } p=\frac{n}{2} \text {. }
$$

Now let us see that $n / 2$ is the minimum genus. If we take any other group $\Gamma$ in the conditions of (3.5), $\Gamma$ would have the signature $\left(g ;+;\left[m_{1} \cdots m_{\tau}\right]\right.$; $\{(-), \ldots,(-)\})$, where $m_{l} \backslash n$, so

$$
p=2+n(2 g-2+k)+n \sum_{\imath \in I}\left(1-\frac{1}{m_{l}}\right) .
$$

If $2 g-2+k>0$, then the genus would be greater than the one we had calculated before; if $2 g-2+k \leq 0$ as $g \geq 0$ and $k \geq 1$, we have that only the following cases can hold: $g=0, k=1 ; g=0, k=2$. If $g=0$, $k=2$, as $|\Gamma|>0$ then $\tau \geq 1$.

$$
p=2+n \sum_{l \in I}\left(1-\frac{1}{m_{l}}\right)>\frac{n}{2} .
$$

If $g=0, k=1$,

$$
p=2-n+n \sum_{i \in I}\left(1-\frac{1}{m_{l}}\right) \text {, }
$$

as $p \geq 3, \tau \geq 2$ necessarily. But $\sum_{l=1}^{\tau}\left(1-1 / m_{l}\right)<2$, since if it is greater or equal, the genus would be greater than the one calculated before. Thus $\tau$ can only be 2 or 3 . In both cases, keeping in mind that l.c.m. $\left(m_{1} \cdots m_{\tau}\right)=n$, one can check easily that the minimum genus one gets is $\geq n / 2$.

If we take an NEC group $\Gamma$ with signature

$$
\left(g ;-;\left[m_{1}, \ldots, m_{\tau}\right] ;\{(-), \ldots,(-)\}\right)
$$


then

$$
p=2+n(g-2+k)+n \sum_{i \in I}\left(1-\frac{1}{m_{i}}\right)
$$

If $g-2+k>0$, then the genus would be greater than the one we had calculated before. If $g-2+k \leq 0$, then, necessarily:

$$
\begin{array}{ll}
g=1, & k=1, \\
g=1, & k=0, \\
g=2, & k=0 .
\end{array}
$$

In the three cases, using Theorem (3.6), we have $p \geq n / 2$.

If $\beta \neq 1$, we consider an NEC group $\Gamma$ of signature

$$
(0 ;+;[n, 2] ;\{(-)\}) \text {. }
$$

This group fulfills the conditions of Theorem (3.5), so

$$
\frac{p-2}{n}=\frac{1}{2}-\frac{1}{n}, \text { i.e. } p=\frac{n}{2}+1 \text {. }
$$

If we take any other group $\Gamma$, by (3.5) and (3.6) and operating in the same way as before, we get that $n / 2+1$ is the minimum genus.

(4.3) TheOREM. Let $n=q_{1}^{r_{1}} \cdots q_{\alpha}^{r_{\alpha}}$, with $q_{1}<q_{2}<\cdots<q_{\alpha}$ being prime numbers and $q_{1} \neq 2$. Then the minimum genus $p$ of a non-orientable Klein surface with group of automorphisms isomorphic to $Z_{n}$ is

$$
\begin{array}{ll}
p=2-q_{1}+n-n / q_{1} & \text { if } r_{1}=1 \\
p=1+n-n / q_{1} & \text { if } r_{1}>1 .
\end{array}
$$

Proof. Similar to the proof of the above theorem, bearing in mind (3.7).

The following corollary has also been obtained by W. Hall in [4]. The corresponding result for orientable Klein surfaces without boundary is due to A. Wiman [10]. 
(4.4) COROllaRy. The maximum order for an automorphism of a non-orientable Klein surface of genus $p \geq 3$ is

$$
\begin{array}{ll}
2 p & \text { if } p \text { is odd, } \\
2(p-1) & \text { if } p \text { is even, }
\end{array}
$$

and it is always reached.

Proof. Given a non-orientable Klein surface of genus $p \geq 3$, we have by Theorems (4.1), (4.2) and (4.3) that the genus $p$ satisfies $p \geq n / 2$, i.e. $2 p \geq n$. If $p=n / 2$, then $n=2$ and $n \neq 4$, so that bound is only reached when $p$ is odd: in fact, given an NEC group $\Gamma$ of signature $(0 ;+;[2, p]$; $\{(-)\})$, by (3.5) there is a non-orientable Klein surface of genus $p$, with a group of automorphisms isomorphic to $Z_{2 p}$.

If $p$ is even, the maximum order for an automorphism is $2(p-1)$, since given an NEC group $\Gamma$ of signature $(0 ;+;[2(p-1), 2] ;\{(-)\})$, by (3.5) there is a non-orientable Klein surface of genus $p$, with a group of automorphisms isomorphic to $Z_{2(p-1)}$.

If $p$ is the topological genus of a compact non-orientable Klein surface without boundary, the algebraic genus is $g=p-1$.

If we express the above corollary in terms of algebraic genus, these bounds are the same as the ones obtained by C. L. May in [6] for the order of an automorphism of an orientable bordered Klein surface.

The author wishes to thank the referee for several helpful comments and suggestions.

\section{REFERENCES}

[1] N. L. Alling, and N. Greenleaf, Foundations of the Theory of Klein Surfaces, Lecture Notes 219, Springer-Verlag (1971).

[2] E. Bujalance, Normal subgroups of NEC group, Math. Z., 178 (1981), 331-341.

[3] _ Proper periods of normal NEC subgroups with even index, to appear in Revista Hispano-Americana.

[4] W. Hall, Automorphisms and coverings of Klein surfaces, Ph.D. Thesis, Southampton (1978).

[5] W. J. Harwey, Cyclic groups of automorphisms of compact Riemann surfaces, Quart J. Math. Oxford, (2) 17 (1966), 86-97.

[6] C. L. May, Cyclic groups of automorphisms of compact bordered Klein surfaces, Houston J. Math., 3, no. 3 (1977), 395-405.

[7] _ _ Automorphisms of compact Klein surfaces with boundary, Pacific J. Math., 59 (1975), 199-210. 
[8] D. Singerman, Automorphisms of compact non-orientable Riemann surface, Glasgow J. Math., 12 (1971), 50-59.

[9] On the structure of non-Euclidean crystallographic groups, Proc. Camb. Phil. Soc., 76 (1974), 223-240.

[10] A. Wiman, Ueber die hyperelliptischen Curven und diejenigen vom Geschlechte $p=3$, welche eindeutigen Transformationen in sich zulassen. Bihan Kongl Svenska Vetenskans-Akademiens Handlingar (Stockholm 1895-6).

Received October 20, 1981 and in revised form June 26, 1982.

DePartamento de Topologia y Geometria

Facultad de Matematicas

UNIVERSIDAD COMPLUTENSE

MADRID, SPAIN 



\title{
PACIFIC JOURNAL OF MATHEMATICS EDITORS
}

\author{
Donald BabBitT (Managing Editor) \\ University of California \\ Los Angeles, CA 90024 \\ Hugo RossI \\ University of Utah \\ Salt Lake City, UT 84112 \\ C. C. Moore and Arthur Ogus \\ University of California \\ Berkeley, CA 94720
}

J. DugundiI

Department of Mathematics

University of Southern California

Los Angeles, CA 90089-1113

R. FinN and H. SAMELSON

Stanford University

Stanford, CA 94305

\section{ASSOCIATE EDITORS}
R. ARENS
E. F. BECKENBACH
B. H. NeUmanN
F. WOLF
K. YosHida
(1906-1982)

\section{SUPPORTING INSTITUTIONS}

\author{
UNIVERSITY OF ARIZONA \\ UNIVERSITY OF BRITISH COLUMBIA \\ CALIFORNIA INSTITUTE OF TECHNOLOGY \\ UNIVERSITY OF CALIFORNIA \\ MONTANA STATE UNIVERSITY \\ UNIVERSITY OF NEVADA, RENO \\ NEW MEXICO STATE UNIVERSITY \\ OREGON STATE UNIVERSITY
}

\author{
UNIVERSITY OF OREGON \\ UNIVERSITY OF SOUTHERN CALIFORNIA \\ STANFORD UNIVERSITY \\ UNIVERSITY OF HAWAII \\ UNIVERSITY OF TOKYO \\ UNIVERSITY OF UTAH \\ WASHINGTON STATE UNIVERSITY \\ UNIVERSITY OF WASHINGTON
}

The Supporting Institutions listed above contribute to the cost of publication of this Journal, but they are not owners or publishers and have no responsibility for its content or policies.

Mathematical papers intended for publication in the Pacific Journal of Mathematics should be in typed form or offset-reproduced (not dittoed), double spaced with large margins. Please do not use built up fractions in the text of the manuscript. However, you may use them in the displayed equations. Underline Greek letters in red, German in green, and script in blue. The first paragraph must be capable of being used separately as a synopsis of the entire paper. In particular it should contain no bibliographic references. Please propose a heading for the odd numbered pages of less than 35 characters. Manuscripts, in triplicate, may be sent to any one of the editors. Please classify according to the scheme of Math. Reviews, Index to Vol. 39. Supply name and address of author to whom proofs should be sent. All other communications should be addressed to the managing editor, or Elaine Barth, University of California, Los Angeles, California 90024.

There are page-charges associated with articles appearing in the Pacific Journal of Mathematics. These charges are expected to be paid by the author's University, Government Agency or Company. If the author or authors do not have access to such Institutional support these charges are waived. Single authors will receive 50 free reprints; joint authors will receive a total of 100 free reprints. Additional copies may be obtained at cost in multiples of 50 .

The Pacific Journal of Mathematics is issued monthly as of January 1966. Regular subscription rate: $\$ 132.00$ a year (6 Vol., 12 issues). Special rate: $\$ 66.00$ a year to individual members of supporting institutions.

Subscriptions, orders for numbers issued in the last three calendar years, and changes of address should be sent to Pacific Journal of Mathematics, P.O. Box 969, Carmel Valley, CA 93924, U.S.A. Old back numbers obtainable from Kraus Periodicals Co., Route 100, Millwood, NY 10546.

The Pacific Journal of Mathematics ISSN $0030-8730$ is published monthly by the Pacific Journal of Mathematics at P.O. Box 969, Carmel Valley, CA 93924. Application to mail at Second-class postage rates is pending at Carmel Valley, California, and additional mailing offices. Postmaster: Send address changes to Pacific Journal of Mathematics, P. O. Box 969, Carmel Valley, CA 93924.

PUBLISHED BY PACIFIC JOURNAL OF MATHEMATICS, A NON-PROFIT CORPORATION

Copyright $\odot 1983$ by Pacific Journal of Mathematics 


\section{Pacific Journal of Mathematics}

Vol. 109, No. $2 \quad$ June, 1983

Tibor Bisztriczky, On the singularities of almost-simple plane curves . . . . 257

Peter B. Borwein, On Sylvester's problem and Haar spaces .............. 275

Emilio Bujalance, Cyclic groups of automorphisms of compact nonorientable Klein surfaces without boundary ............... 279

Robert Jay Daverman and John J. Walsh, Acyclic decompositions of

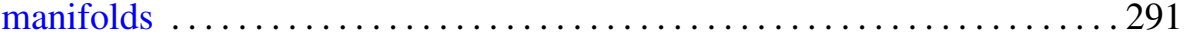

Lester Eli Dubins, Bernstein-like polynomial approximation in higher

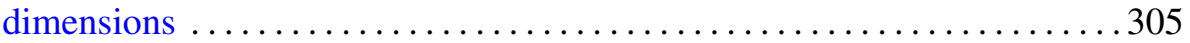

Allan L. Edelson and Jerry Dee Schuur, Nonoscillatory solutions of

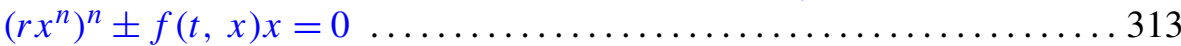

Akira Endô, On units of pure quartic number fields ................. 327

Hector O. Fattorini, A note on fractional derivatives of semigroups and

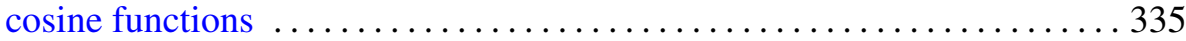

Ronald Fintushel and Peter Sie Pao, Circle actions on homotopy spheres with codimension 4 fixed point set ........................ 349

Stephen Michael Gagola, Jr., Characters vanishing on all but two

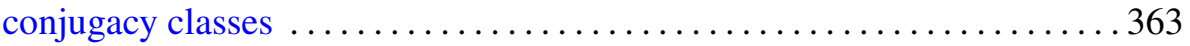

Saverio Giulini, Singular characters and their $L^{p}$ norms on classical Lie

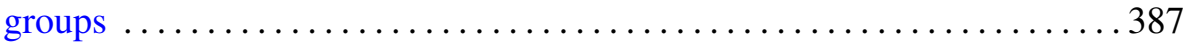

Willy Govaerts, Locally convex spaces of non-Archimedean valued continuous functions

Wu-Chung Hsiang and Bjørn Jahren, A remark on the isotopy classes of diffeomorphisms of lens spaces $\ldots . \ldots \ldots \ldots \ldots \ldots$

Hae Soo Oh, Compact connected Lie groups acting on simply connected 4-manifolds

Frank Okoh and Frank A. Zorzitto, Subsystems of the polynomial system

Knut Øyma, An interpolation theorem for $H_{E}^{\infty}$

Nikolaos S. Papageorgiou, Nonsmooth analysis on partially ordered vector spaces. II. Nonconvex case, Clarke's theory ........ 